\title{
Vortex-Based Spin Transfer Oscillator Compact Model for IC Design
}

\author{
Nicolas Locatelli*, Damir Vodenicarevic*, Weisheng Zhao*, Jacques-Olivier Klein* \\ Julie Grollier ${ }^{\dagger}$ and Damien Querlioz* \\ *Institut d'Électronique Fondamentale, Univ. Paris-Sud, CNRS, Orsay 91405, France \\ †Unité Mixte de Physique CNRS/Thales, 1 av A. Fresnel, 91767 Palaiseau, France, \\ and Univ Paris Sud, 91405 Orsay, France
}

\begin{abstract}
Spintronic oscillators are nanodevices that are serious candidates for $\mathrm{CMOS}$ integration due to their compactness and easy frequency tunability. Among them vortex-based oscillators appear as one of the most promising technology because of their lower power supply and higher quality factors. To assess their potential in circuits and systems, compact models describing their behavior are necessary. In this work, we propose an implementation of a spintronic nano-oscillator (STNO) model for integrated circuit (IC) architectures design. The modeled device is a vortex-based magnetic oscillator demonstrating selfsustained magnetization oscillations under current bias, inducing alternating voltage across the device. This model describes the coupled electrical and magnetic behavior of the device, taking into account phase and amplitude noises associated with thermal fluctuations. Compatibility with commercial CMOS design kits is demonstrated, and an implementation in a CMOS circuit is proposed for AC signal generation. These results will allow to develop and evaluate innovative hybrid STNO/CMOS systems and their potential to efficiently complement existing full-CMOS technologies.
\end{abstract}

\section{INTRODUCTION}

The recent development of spin transfer torque magnetic random access memories (STT-MRAMs) unveiled the potential of CMOS integration of spintronic devices into innovative architectures [1]-[3]. Among the large diversity of spintronic elements, other devices also hold potential for applications [4], [5]. Magnetic tunnel junctions (MTJs), the basic cell of the MRAM, can also be engineered to achieve self-sustained magnetic oscillations under DC current bias, and the spintransfer effect thereof. These magnetization oscillations are then transcribed into resistance oscillations through the tunnel magneto-resistance (TMR) effect, generating oscillations of the device voltage at frequencies typically ranging from $100 \mathrm{MHz}$ to 10 's of $\mathrm{GHz}$ depending on the device geometry and magnetic parameters.

The small size of the so-called spin-transfer nanooscillators (STNOs) and their ability to tune their frequency with the supply current amplitude are strong assets for their integration as radio-frequency sources in communication systems [6]-[9]. Also recently, those STNOs were proposed as key-elements for the design of associative memories architectures based on highly connected oscillators networks [10], [11], expected to yield high computing capability notably for pattern recognition. This proposal is based on the demonstrated ability of the STNOs to achieve mutual synchronization with one or more interacting neighbors under adequate conditions [12][15]. For all these purposes, the development of compact SPICE-compatible models for integration in CMOS design platforms is a prerequisite.

Previous works on compact modeling of spin transfer oscillators have focused on a certain type of oscillators based on the excitation of a uniform magnetic mode [16], [17], and models were integrated under the strong assumption of macrospin behavior. The present work focuses on STNOs based on the excitation of a magnetic vortex. This type of oscillators has experimentally demonstrated high quality factors as compared to other types of STNOs [18], [19], as well as a capabilities to tune its frequency with the bias current amplitude and to synchronize to an external excitation from an AC-source [20], [21] or to mutually-synchronize to coupled oscillators [15].

We propose a Verilog-A implementation of the physical model describing the self-sustained vortex oscillations in a pillar STNO for a use in IC design of hybrid MTJ/CMOS systems. To correctly describe the oscillator behavior at working temperature, our model implementation includes parameters non-linearities [22] as well as the phase and amplitude noise effects under thermal fluctuations [23], [24]. These factors can indeed be of major importance to accurately describe the synchronization phenomenon.

After introducing the device and the associated magnetic and electrical equations, we present the model implementation under the Cadence platform and confront the obtained simulations to previously obtained experimental results. Finally, we demonstrate the compatibility of our model with a CMOS design kit for IC design by simulating an integrated current source for biasing and synchronization of the STNO.

\section{The SPIN-Transfer Vortex Oscillator MOdEL}

The device under consideration is a nanopillar hybrid spin transfer vortex based oscillator, as introduced in [25] and presented in Figure 1(a). It is made of a layered stack, first composed of a metallic spin-valve structure including a perpendicularly magnetized ferromagnetic layer, a non-magnetic spacer and a central ferromagnetic layer. The latter is also part of a magnetic tunnel junction, composed of an insulating barrier and an in-plane magnetized ferromagnetic layer. The magnetizations of both perpendicular and in-plane magnetized ferromagnets are considered pinned. Dimensions of the pillar and particularly the aspect ratio of the central ferromagnet are 
(a)
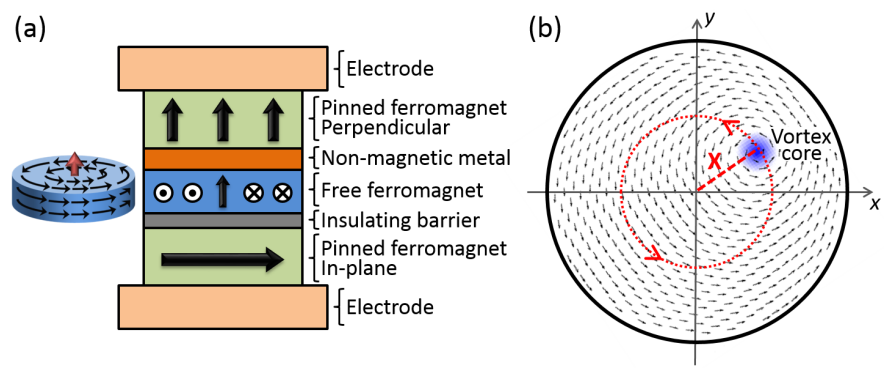

Fig. 1. (a) Sample description. Arrows illustrate the magnetization distribution in ferromagnets. (b) Illustration of the vortex magnetization dynamics. Arrows and color scale respectively describe the in-plane and out-of-plane magnetization distribution.

chosen so that its remanent magnetic state is a vortex [26]. Such magnetic state corresponds to the magnetization curling in the layer's plane and popping out-of-plane in the center region called the vortex core (see Figure 1). The studied mode of the vortex, called "gyrotropic mode", corresponds to a circular displacement of the vortex core around the center of the magnetic dot [26], [27] as illustrated in Figure 1(b).

As current flows through the device, the two pinned layers act as spin-current sources generating a spin-transfer effect on the vortex. As demonstrated in previous works, a source of perpendicular spin polarization is mandatory to start and sustain the vortex precession [28], [29]. On the other hand, the MTJ with in-plane magnetized layer is needed to convert the magnetization oscillations into resistance oscillations [25], and plays a major role in the synchronization process by providing an in-plane component to the current spin polarization [30]. Under sufficient current bias, the device then demonstrates permanent resistance oscillations and a resulting $\mathrm{AC}$ voltage appears across the device.

Because the vortex gyrotropic mode is isolated from other higher frequency modes, the self-sustained oscillations under spin-transfer effect can be properly described by a collectivevariable equation, called the Thiele equation [26], [27], where the global dynamics is simply described through the evolution of the position of the magnetic vortex core. This equation was recently extended to include the spin-transfer terms [22], [28], [29]. The device is then modeled according to the following equations, where $\mathbf{X}=\rho e^{i \chi}$ is the vortex core complex coordinate (see Figure 1(b)):

$$
\begin{aligned}
& i G \dot{\mathbf{X}}=-D(\rho) \dot{\mathbf{X}}-k(\rho) \mathbf{X}+i \kappa_{\perp} I \mathbf{X}+\kappa_{\|} I+F_{n o i s e}(t) \\
& R=R_{\text {min }}\left(1+\xi C \mathrm{TMR}_{\%} \frac{\operatorname{Im}(\mathbf{X})}{r}\right)
\end{aligned}
$$

Equation 1 is the modified Thiele equation describing the dynamics of the vortex core, accounting for conservative and dissipative terms. The left hand side gyrotropic term is equal to the sum of the effective forces acting on the vortex core : the effective damping force, the spring-like confinement force, and the effective spin-transfer forces induced respectively by the perpendicular and the in-plane spin polarizations of the current. Equation 2 relates the value of the resistance to the position of the vortex core. All the terms appearing in these equations are derived from geometrical and magnetic parameters. Their
TABLE I. GEOMETRICAL AND MAGNETIC PARAMETERS FOR THE FREE FERROMAGNETIC LAYER AND SPIN POLARIZED CURRENT.

\begin{tabular}{|c|c|l|}
\hline Symbol & Expression & Description \\
\hline$r$ & $90 \mathrm{~nm}$ & Pillar radius \\
$t$ & $10 \mathrm{~nm}$ & Free layer thickness \\
$\mu_{0} M_{S}$ & $1.0 \mathrm{~T}$ & Free layer saturation magnetization \\
$\alpha$ & 0.01 & Free layer Gilbert damping \\
$L_{e}$ & $6 \mathrm{~nm}$ & Free layer exchange length \\
$p_{\perp}$ & $60 \%$ & Current spin polarization along perpendicular z-axis \\
$p_{\|}$ & $100 \%$ & Current spin polarization along in-plane x-axis \\
\hline
\end{tabular}

TABLE II. PARAMETERS FOR THE THIELE EQUATION MODELING THE VORTEX GYROTROPIC MOTION. $\gamma$ IS THE ELECTRON GYROMAGNETIC RATIO, $\mu_{B}$ IS THE BOHR MAGNETON, $\mu_{0}$ IS THE VACUUM PERMEABILITY,

\begin{tabular}{|c|c|c|c|}
\hline Symbol & Expression & Description & Units \\
\hline$G$ & $2 \pi t M_{S} / \gamma$ & Gyrovector amplitude & $N . s / m$ \\
\hline$\eta$ & $\frac{1}{2} \ln \left(\frac{r}{4 L_{e}}\right)-\frac{1}{8}$ & $1^{s t}$ order damping & unitless \\
\hline$\eta^{\prime}$ & $1 / 12$ & $2^{n d}$ order damping & unitless \\
\hline$D(\rho)$ & $\alpha G\left(\eta+\eta^{\prime}\left(\frac{\rho}{r}\right)^{2}\right)$ & Non-linear damping & $N . s / m$ \\
\hline$k_{m s}$ & $\frac{10}{9} \mu_{0} M_{S}^{2} t^{2} / r$ & $\begin{array}{l}1^{s t} \text { order magneto-static } \\
\text { confinement }\end{array}$ & $N / m$ \\
\hline$k_{m s}^{\prime}$ & $k_{m s} / 4$ & $\begin{array}{l}2^{n d} \text { order magneto-static } \\
\text { confinement }\end{array}$ & $N / m$ \\
\hline$k_{O e}$ & $0.85 \mu_{0} M_{S} t /(\pi r)$ & $\begin{array}{l}1^{\text {st }} \text { order Oersted field } \\
\text { confinement }\end{array}$ & $N . m / A$ \\
\hline$k_{O e}^{\prime}$ & $-k_{O e} / 2$ & $\begin{array}{l}2^{\text {nd }} \text { order Oersted field } \\
\text { confinement }\end{array}$ & $N . m / A$ \\
\hline$k(\rho)$ & $\begin{array}{l}\left(k_{m s}+k_{O e} I\right) \\
\quad+\left(k_{m s}^{\prime}+k_{O e}^{\prime} I\right)\left(\frac{\rho}{r}\right)^{2}\end{array}$ & $\begin{array}{l}\text { Non-linear confinement } \\
\text { coefficient }\end{array}$ & $N / m$ \\
\hline$a_{J}$ & $g \mu_{B} /\left(2 t e M_{S}\right)$ & Spin transfer efficiency & $m^{2} / A / s$ \\
\hline$\kappa_{\perp}$ & $a_{J} p_{\perp} G /\left(2 \pi r^{2}\right)$ & Orthogonal spin transfer & $N . m / A$ \\
\hline$\kappa_{\|}$ & $a_{J} p_{\|} G L_{e} \ln (2) /\left(\pi r^{2}\right)$ & In-plane spin transfer & $N \cdot m^{2} / A$ \\
\hline$\xi$ & $2 / 3$ & $\begin{array}{l}\text { Average magnetization to } \\
\text { vortex displacement ratio }\end{array}$ & unitless \\
\hline$\omega\left(s^{2}\right)$ & $\begin{array}{l}k(\rho) / G \\
\quad+\alpha \eta(\rho) \kappa_{\perp} I / G\end{array}$ & $\begin{array}{l}\text { Instantaneous frequency } \\
\text { (amplitude dependent) }\end{array}$ & $\mathrm{rad} / \mathrm{s}$ \\
\hline$v_{c}$ & $\frac{1}{3} L_{e} \gamma \mu_{0} M_{S}$ & Critical vortex velocity & $\mathrm{m} / \mathrm{s}$ \\
\hline
\end{tabular}
$g=2$ IS THE LANDE FACTOR AND $e$ IS THE ELECTRON CHARGE.

expressions are gathered in table II. Additionally, on the right-hand side, a phenomenological stochastic force $F_{\text {noise }}(t)$ has been added, describing the action of thermal fluctuations according to fluctuation-dissipation theorem, and derived in the framework of the classical nonlinear oscillators theory [23], [24], [31]. Finally, oscillations amplitude limitation is ruled by the existence of a critical velocity $v_{c}$ for the vortex core that will trigger a polarity switching and subsequently stop the self-sustained oscillations [29].

\section{MODEL IMPLEMENTATION}

The described physical model was implemented in VerilogA language under the Cadence Spectre simulator. Simulations were conducted for a $2 r=180 \mathrm{~nm}$ diameter device, with a $t=10 \mathrm{~nm}$ thick free $\mathrm{NiFe}$ layer whose magnetic parameters are gathered in table I. The minimum resistance was chosen to be $R_{\text {min }}=130 \Omega$ with a $\mathrm{TMR}$ ratio $\mathrm{TMR}_{\%}=70 \%$ for the tunnel junction, in agreement with previously fabricated devices. A $p_{\perp}=60 \%$ perpendicular polarization (from the the spin-valve) and an ideal $p_{\|}=100 \%$ in-plane polarization (from the MTJ) were assumed. These parameters correspond to a critical $220 \mu \mathrm{A}$ current to start the self-sustained oscillations.

The model was tested under constant current bias for transient simulations, in both cases of noise-free and noisy 
oscillator. The vortex core position was initiated in a random position in the vicinity of the ferromagnet center, allowing the observation of a transitory regime where the oscillations amplitude increases up to its stable value, starting the steady regime of oscillations.

A major criteria for the validation of our model was the stability of the noise-free auto-oscillations frequency and amplitude in the steady-state regime and their independence to the simulation time step. Due to different time-scales for the evolution of the phase and the amplitude of oscillations, we found that the compact model is largely improved by implementing equation (1) under polar coordinates instead of Cartesian. We then evaluated that to ensure a steady-state frequency stability, a reasonable criteria was ensuring that the time step for the simulation verifies $\Delta t<T(\rho) / 50$ where $T(\rho)=2 \pi / \omega(\rho)$ is the instantaneous oscillation period of the vortex. We enforce this minimum time step thanks to a bound_step VerilogA call within the compact model.

The thermal fluctuation term was implemented through a random Gaussian drawing at each integration step of the differential equation. Compatibility of this model with fluctuationdissipation theory was then verified by evaluating the average fluctuating energy at equilibrium. At zero bias, this energy $\langle\epsilon\rangle=\left\langle\frac{1}{2} k(\rho) \rho^{2}\right\rangle$ was verified to be equal to the thermal energy $k_{B} T$.

$10 \mu$ s simulations of a single oscillator on a $3.20 \mathrm{GHz}$ CPU were obtained in respectively $9.43 \mathrm{~s}$ and $9.81 \mathrm{~s}$ for the noisefree and room-temperature noisy oscillator. In Figures 2(a) and (b), we present the temporal evolution of the device voltage under a constant $260 \mu \mathrm{A}$ current bias in both cases. The observed auto-oscillation frequency is $617.8 \mathrm{MHz}$, in good agreement with the expected frequency for the chosen device dimensions [26]. As it appears on the corresponding spectrum on Figure 2(c), fluctuations in the noisy oscillator translate into phase noise that broadens the spectrum peak, accounting for the non-zero peak linewidth observed experimentally [25]. A $14.6 \mathrm{MHz}$ linewidth is measured, independent on the time step, in good agreement with the $14.0 \mathrm{MHz}$ theoretically predicted linewidth [23].

\section{COMPATIBILITY WITH CMOS DESIGN KIT}

We have tested the compatibility of our model with a commercial 28-nm CMOS design kit by building a MOSFETbased supply for the STNO (see Figure 3). The circuit is a voltage controlled current source. A cascode architecture is implemented to reduce the impact of voltage oscillations across the STNO on the current bias. The frequency of the autooscillator is then controlled by the gate voltage of $T 1$ transistor, as summarized in Figure 3.

The circuit is designed so that a zero command voltage corresponds to below threshold current bias. Self-sustained oscillations appear when a $V_{c d e}>10 \mathrm{mV}$ voltage is applied, and their amplitude keeps increasing as $V_{c d}$ is swept up to $180 \mathrm{mV}$ above which the critical vortex speed is reached and oscillations are terminated. Because both current bias and resistance oscillations amplitude are different for every frequency value, it is important to note that the AC voltage amplitude is also varying as the command voltage is swept.

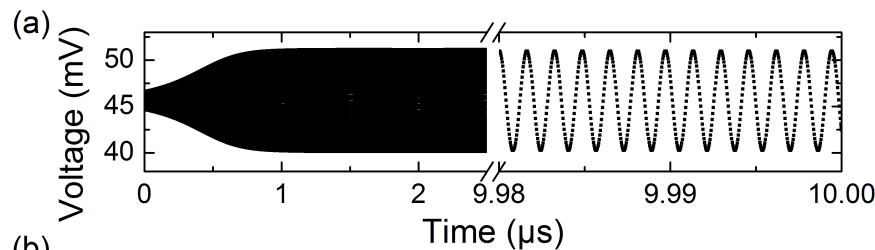

(b)

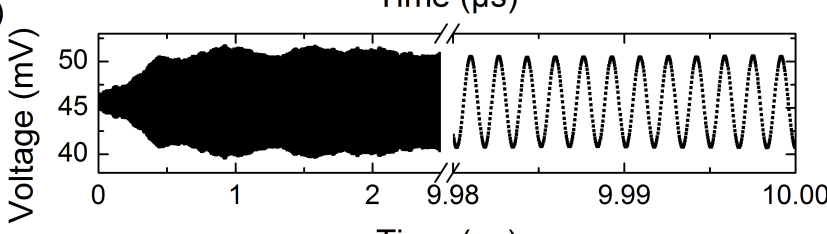

(c)

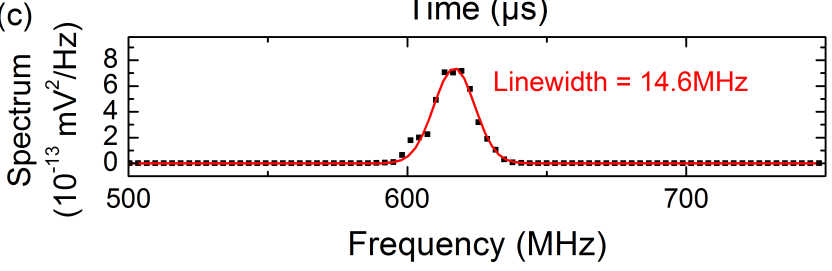

Fig. 2. (a) Simulated voltage versus time for a noise-free oscillator under a constant $260 \mu \mathrm{A}$ bias. (b) Simulated voltage versus time and (c) associated spectrum for a noisy oscillator under a constant $260 \mu \mathrm{A}$ bias. Full Width at Half-Maximum is determined by fitting the spectrum peak with a Gaussian function.
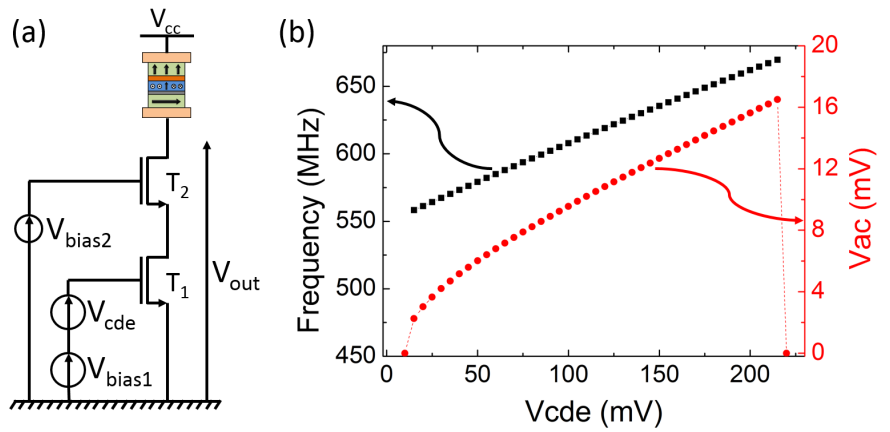

Fig. 3. (a) MOSFET-based control circuit for the spin transfer oscillator, simulated using a commercial 28nm-node design kit. (b) Frequency and peakto-peak amplitude of the output voltage $V_{\text {out }}$ versus command voltage.

As it appears on Figure 3, the voltage amplitude varies between 0 and $15 \mathrm{mV}$.

Spin transfer nano-oscillators show the ability to respond to an AC-current excitation by synchronizing to the input signal for sufficient amplitude and if its frequency is close enough to the auto-oscillation frequency [20], [21]. To test the ability of the implemented model to describe this ability, we simulated the response of the STNO when a harmonic AC-voltage is added to the command voltage. The simulation was run for a $V_{c d e}=120 \mathrm{mV}$ corresponding to a $261 \mu \mathrm{A}$ current bias and a frequency $619 \mathrm{MHz}$, to which an AC-signal at a frequency $609 \mathrm{MHz}$ was added. We then found that a minimum $100 \mathrm{mV}$ amplitude is necessary for the AC-input for the STNO to shift its frequency and synchronize, corresponding to an AC-current amplitude $i_{A C}=27.2 \mu \mathrm{A}$.

\section{CONCLUSION AND PERSPECTIVES}

We proposed and tested a Verilog-A implementation of a vortex-based spin transfer oscillator compact model for CMOS 
integration. This model accounts for the non-linear behavior of the oscillator as well as the thermally induced fluctuations of its phase and amplitude. It has been found to describe the frequency tunability with bias current as well as the synchronization ability of the oscillators, in accordance with experimental results.

After being tested under ideally constant current bias, the device model was successfully inserted in a MOSFET-based circuit for supply and frequency control of the STNO using a commercial CMOS design kit. We then demonstrated the compatibility of the proposed model for integration in IC design platforms.

This model can readily be used for applications of STNOs which have been proposed for telecommunications. Additionally, the use of STNOs and of their ability to synchronize have been brought up as a serious candidate for the design of innovative processing architectures, especially for data recognition [10], [11]. As vortex-based spin transfer oscillators are promising candidates for the study and proof of concept for these architecture, this model will be useful for IC design of such future hybrid MTJ/CMOS systems.

\section{ACKNOWLEDGMENT}

The authors acknowledge E. Grimaldi and J.-V. Kim for fruitful discussions. This work was supported by the ANR COGNISPIN (ANR-13-JS03-0004-01) and the FP7 ICT BAMBI (FP7-ICT-2013-C) projects, and by Labex NanoSaclay (Chaire fiabilité des nanos).

\section{REFERENCES}

[1] A. V. Khvalkovskiy, D. Apalkov, S. Watts, R. Chepulskii, R. S. Beach et al., "Basic principles of STT-MRAM cell operation in memory arrays," Jour. of Phys. D: Appl. Phys., vol. 46, no. 7, p. 074001, Feb. 2013, 00016.

[2] W. Zhao, J.-O. Klein, Z. Wang, Y. Zhang, N. Ben Romhane et al., "Spinelectronics based logic fabrics," in 2013 IFIP/IEEE 21st International Conference on VLSI-SoC, Oct. 2013, pp. 174-179, 00003.

[3] Y. Zhang, W. Zhao, J.-O. Klein, W. Kang, D. Querlioz et al., "Spintronics for low-power computing," in Design, Automation and Test in Europe Conference and Exhibition, 2014, Mar. 2014, pp. 1-6, 00001.

[4] G. Prenat, B. Dieny, W. Guo, M. El Baraji, V. Javerliac et al., "Beyond MRAM, CMOS/MTJ integration for logic components," IEEE Trans. Magn., vol. 45, no. 10, pp. 3400-3405, Oct. 2009.

[5] N. Locatelli, V. Cros, and J. Grollier, "Spin-torque building blocks," Nature Materials, vol. 13, no. 1, pp. 11-20, Jan. 2014.

[6] P. Villard, U. Ebels, D. Houssameddine, J. Katine, D. Mauri et al., "A GHz spintronic-based RF oscillator," IEEE Journal of Solid-State Circuits, vol. 45, no. 1, pp. 214-223, Jan. 2010.

[7] O. Prokopenko, E. Bankowski, T. Meitzler, V. Tiberkevich, and A. Slavin, "Spin-torque nano-oscillator as a microwave signal source," IEEE Magnetics Letters, vol. 2, pp. 3000 104-3000 104, 2011.

[8] E. Grimaldi, R. Lebrun, A. Jenkins, A. Dussaux, J. Grollier et al., "Spintronic nano-oscillators: Towards nanoscale and tunable frequency devices," in 2014 IEEE International Frequency Control Symposium, pp. 1-6.

[9] H. S. Choi, S. Y. Kang, S. J. Cho, I.-Y. Oh, M. Shin et al., "Spin nanooscillator-based wireless communication," Scientific Reports, vol. 4 2014.

[10] S. P. Levitan, Y. Fang, J. A. Carpenter, C. N. Gnegy, N. S. Janosik et al., "Associative processing with coupled oscillators," in 2013 IEEE International Symposium on Low Power Electronics and Design, Piscataway, NJ, USA, 2013, pp. 235-235.
[11] G. Csaba, M. Pufall, D. Nikonov, G. Bourianoff, A. Horvath et al., "Spin torque oscillator models for applications in associative memories," in 2012 13th International Workshop on CNNA, 2012, pp. 1-2, 00005.

[12] S. Kaka, M. R. Pufall, W. H. Rippard, T. J. Silva, S. E. Russek et al., "Mutual phase-locking of microwave spin torque nano-oscillators," Nature, vol. 437, no. 7057, pp. 389-392, Sep. 2005.

[13] F. B. Mancoff, N. D. Rizzo, B. N. Engel, and S. Tehrani, "Phaselocking in double-point-contact spin-transfer devices," Nature, vol. 437, no. 7057, pp. 393-395, Sep. 2005.

[14] J. Grollier, V. Cros, and A. Fert, "Synchronization of spin-transfer oscillators driven by stimulated microwave currents," Phys. Rev. B, vol. 73, no. 6, p. 060409, 2006.

[15] A. D. Belanovsky, N. Locatelli, P. N. Skirdkov, F. A. Araujo, K. A. Zvezdin et al., "Numerical and analytical investigation of the synchronization of dipolarly coupled vortex spin-torque nano-oscillators," Appl. Phys. Lett., vol. 103, no. 12, p. 122405, Sep. 2013.

[16] M. Stan, M. Kabir, J. Lu, and S. Wolf, "Nano-pattemed coupled spin torque nano oscillator (STNO) arrays ; a potentially disruptive multipurpose nanotechnology," in IEEE 11th International New Circuits and Systems Conference (NEWCAS), Jun. 2013, pp. 1-4.

[17] G. Csaba, M. Pufall, W. Rippard, and W. Porod, "Modeling of coupled spin torque oscillators for applications in associative memories," in 12th IEEE Conference on Nanotechnology, 2012, pp. 1-4.

[18] V. S. Pribiag, I. N. Krivorotov, G. D. Fuchs, P. M. Braganca, O. Ozatay et al., "Magnetic vortex oscillator driven by d.c. spin-polarized current," Nature Physics, vol. 3, no. 7, pp. 498-503, May 2007.

[19] A. Dussaux, B. Georges, J. Grollier, V. Cros, A. V. Khvalkovskiy et al., "Large microwave generation from current-driven magnetic vortex oscillators in magnetic tunnel junctions," Nature Communications, vol. 1, p. 8, Apr. 2010.

[20] A. Dussaux, A. V. Khvalkovskiy, J. Grollier, V. Cros, A. Fukushima et al., "Phase locking of vortex based spin transfer oscillators to a microwave current," Appl. Phys. Lett., vol. 98, no. 13, pp. 132506132 506-3, Mar. 2011.

[21] R. Lehndorff, D. E. Bürgler, C. M. Schneider, and Z. Celinski, "Injection locking of the gyrotropic vortex motion in a nanopillar," Appl. Phys. Lett., vol. 97, no. 14, pp. 142 503-142 503-3, Oct. 2010.

[22] A. Dussaux, A. V. Khvalkovskiy, P. Bortolotti, J. Grollier, V. Cros et al., "Field dependence of spin-transfer-induced vortex dynamics in the nonlinear regime," Phys. Rev. B, vol. 86, no. 1, p. 014402, 2012.

[23] V. S. Tiberkevich, A. N. Slavin, and J.-V. Kim, "Temperature dependence of nonlinear auto-oscillator linewidths: Application to spin-torque nano-oscillators," Phys. Rev. B, vol. 78, no. 9, p. 092401, 2008.

[24] E. Grimaldi, A. Dussaux, P. Bortolotti, J. Grollier, G. Pillet et al., "Response to noise of a vortex based spin transfer nano-oscillator," Phys. Rev. B, vol. 89, no. 10, p. 104404, Mar. 2014, 00000.

[25] A. Dussaux, E. Grimaldi, B. R. Salles, A. S. Jenkins, A. V. Khvalkovskiy et al., "Large amplitude spin torque vortex oscillations at zero external field using a perpendicular spin polarizer," Appl. Phys. Lett., vol. 105, no. 2, p. 022404, Jul. 2014

[26] K. Y. Guslienko, "Magnetic vortex state stability, reversal and dynamics in restricted geometries," J. Nanosci and Nanotechno., vol. 8, no. 6, pp. 2745-2760, 2008

[27] A. A. Thiele, "Steady-state motion of magnetic domains," Phys. Rev Lett., vol. 30, no. 6, pp. 230-233, 1973.

[28] Q. Mistral, M. van Kampen, G. Hrkac, J.-V. Kim, T. Devolder et al., "Current-driven vortex oscillations in metallic nanocontacts," Phys. Rev. Lett., vol. 100, no. 25, p. 257201, 2008.

[29] A. V. Khvalkovskiy, J. Grollier, A. Dussaux, K. A. Zvezdin, and V. Cros, "Vortex oscillations induced by spin-polarized current in a magnetic nanopillar: Analytical versus micromagnetic calculations," Phys. Rev. $B$, vol. 80, no. 14, p. 140401, Oct. 2009.

[30] A. V. Khvalkovskiy, J. Grollier, N. Locatelli, Y. V. Gorbunov, K. A. Zvezdin et al., "Nonuniformity of a planar polarizer for spin-transferinduced vortex oscillations at zero field," Appl. Phys. Lett., vol. 96, no. 21, pp. $212507-212507-3$, May 2010.

[31] T. Kamppeter, F. Mertens, A. Snchez, A. Bishop, F. Domnguez-Adame et al., "Finite temperature dynamics of vortices in the two dimensional anisotropic heisenberg model," Eur. Phys. J. B, vol. 7, no. 4, pp. 607$618,1999$. 